\title{
Parameter Estimation Techniques for a Polarization Hysteresis Model
}

\author{
Ralph C. Smith* and Andrew Hatch ${ }^{\dagger}$ \\ Center for Research in Scientific Computation \\ Department of Mathematics \\ North Carolina State University \\ Raleigh, NC 27695
}

\begin{abstract}
This paper focuses on the development of parameter estimation techniques for models quantifying hysteresis and constitutive nonlinearities in ferroelectric materials. These models are formulated as integral equations with known kernels and unknown densities to be identified through least squares fit to data. Due to the compactness of the integral operators, the resulting discretized models inherit ill-posedness which often must be accommodated through regularization. The accuracy of regularized finite-dimensional models is illustrated through comparison with experimental data.
\end{abstract}

Keywords: Hysteresis model, compact operator, parameter estimation, regularization

\section{Introduction}

Piezoceramic (PZT), magnetostrictive and shape memory alloy (SMA) compounds exhibit hysteresis and constitutive nonlinearities which must be incorporated in models and model-based control algorithms to achieve the novel performance capabilities offered by the materials. A number of modeling strategies for these compounds have been proposed but three stand out in the sense that they provide unified frameworks for characterizing hysteresis in ferroelectric, ferromagnetic and ferroelastic materials, which are collectively referred to as ferroic compounds. These three approaches are the following: (i) homogenized free energy models [9, 13, 18], (ii) Preisach formulations $[1,2,12,19]$, and (iii) domain wall models $[6,8,11,14,15]$. The first two are formulated as integral equations whereas the domain wall models are typically posed as differential equations.

To simplify the discussion, we focus on the estimation of parameters in the homogenized free energy model characterizing the hysteretic field-polarization relation for ferroelectric materials. It is demonstrated in [17] that this framework can be used to characterize hysteresis in general ferroic compounds so analogous techniques can be employed for ferromagnetic and ferroelastic materials. It is illustrated in [16] that this framework provides an energy basis for certain extended Preisach formulations, and compactness results analogous to those established here are proven for classical Preisach operators by Iyer and Shirley [7]. The reader is also referred to [5] for details regarding the recursive identification of Preisach density functions.

The model is summarized in Section 2 and compactness of the integral operator is established in Section 3. Parameter identification algorithms are summarized in Section 4 and illustrated in Section 5 in the context of characterizing PZT5H data.

\footnotetext{
*Email: rsmith@eos.ncsu.edu; Telephone: 919-515-7552

${ }^{\dagger}$ Email: aghatch@eos.ncsu.edu; Telephone: 919-515-7241
} 


\section{Polarization Model}

It is demonstrated in [18] that the hysteretic relation between the electric field $E$ and polarization $P$ in ferroelectric materials can be characterized by the model

$$
[P(E)](t)=\int_{0}^{\infty} \int_{-\infty}^{\infty} \nu\left(E_{c}, E_{e}\right)\left[\bar{P}\left(E_{e}+E ; E_{c}, \xi\right)\right](t) d E_{e} d E_{c}
$$

where $\nu$ is a material-dependent parameter, $\bar{P}$ is the piecewise linear kernel or hysteron depicted in Figure 1 , and $E_{c}, E_{e}$ respectively denote local coercive and effective fields. The kernel has the general form

$$
\bar{P}\left(E_{e}+E ; E_{c}, \xi\right)=\frac{E}{\eta}+P_{R} \delta\left(E ; E_{c}, E_{e}\right)
$$

where $\xi$ delineates initial dipole orientations. The parameter $\delta$ has a value of 1 for positively oriented dipoles and -1 for negative orientations. More rigorously, $\bar{P}$ can be specified as

$$
\left[\bar{P}\left(E ; E_{c}, \xi\right)\right](t)= \begin{cases}{\left[\bar{P}\left(E ; E_{c}, \xi\right)\right](0)} & , \tau(t)=\emptyset \\ \frac{E}{\eta}-P_{R} & , \tau(t) \neq \emptyset \text { and } E(\max \tau(t))=-E_{c} \\ \frac{E}{\eta}+P_{R} & , \tau(t) \neq \emptyset \text { and } E(\max \tau(t))=E_{c}\end{cases}
$$

where

$$
\left[\bar{P}\left(E ; E_{c}, \xi\right)\right](0)= \begin{cases}\frac{E}{\eta}-P_{R} & , E(0) \leq-E_{c} \\ \xi & ,-E_{c}<E(0)<E_{c} \\ \frac{E}{\eta}+P_{R} & , E(0) \geq E_{c}\end{cases}
$$

defines the initial states of the kernel and

$$
\tau(t)=\left\{t \in\left(0, T_{f}\right] \mid E(t)=-E_{c} \text { or } E(t)=E_{c}\right\}
$$

designates the set of switching times.

The density is assumed to have the product formulation $\nu\left(E_{c}, E_{e}\right)=\nu_{1}\left(E_{c}\right) \nu_{2}\left(E_{e}\right)$ where $\nu_{1}$ and $\nu_{2}$ satisfy the physical conditions

$$
\begin{array}{ll}
\text { (i) } & \nu_{1}(x) \text { defined for } x>0, \\
\text { (ii) } & \nu_{2}(-x)=\nu_{2}(x) \\
\text { (iii) } & \left|\nu_{1}(x)\right| \leq c_{1} e^{-a_{1}\left(x-b_{1}\right)} \\
& \left|\nu_{2}(x)\right| \leq c_{2} e^{-a_{2} x}
\end{array}
$$

for positive $c_{1}, a_{1}, b_{1}, c_{2}, a_{2}$. The goal in the parameter identification problem is to estimate $\nu$ given data measurements $\left\{\left(\widehat{E}_{k}, \widehat{P}_{k}\right)\right\}, k=1, \ldots, N_{d}$.

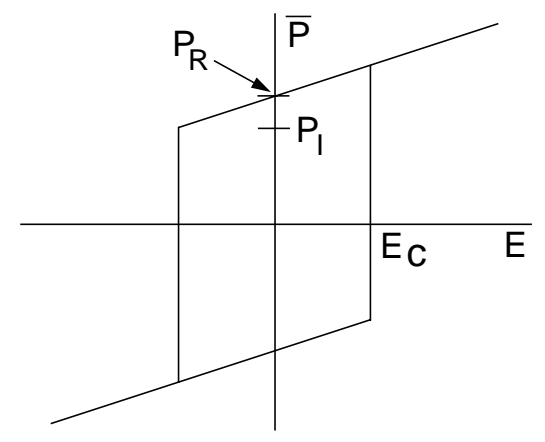

Figure 1: Piecewise linear kernel $\bar{P}$. 
By invoking the physical decay criteria, (1) can be approximated to arbitrary accuracy by consideration of

$$
[P(E)](t)=\iint_{\Omega_{2}} \nu\left(E_{c}, E_{e}\right)\left[\bar{P}\left(E+E_{e} ; E_{c}, \xi\right)\right](t) d E_{e} d E_{c}
$$

on the compact domain

$$
\Omega_{2}=\left\{\left(E_{c}, E_{e}\right) \in \mathbb{R}_{+} \times \mathbb{R} \mid \nu\left(E_{c}, E_{e}\right) \geq \epsilon\right\} .
$$

Furthermore, we let the minimum and maximum admissible input fields be denoted $E_{\min }$ and $E_{\max }$ and define

$$
\Omega_{1}=\left[E_{\min }, E_{\max }\right]
$$

We consider parameters $q=\nu$ in the parameter space

$$
\mathcal{Q}=L^{2}\left(\Omega_{2}\right)
$$

and define the observation operator $\mathcal{C} P=P(E)$ on the observation space

$$
\mathcal{Y}=L^{2}\left(P_{\min }, P_{\max }\right) .
$$

The polarization model (4) can then be formulated as

$$
y(E)=\mathcal{K} q(E)
$$

where

$$
E \in C\left[\Omega_{1}\right] \subset L^{2}\left(\Omega_{1}\right)
$$

and the parameter-to-observation operator $\mathcal{K}$ is defined by

$$
\mathcal{K} q=\mathcal{C} \iint_{\Omega_{2}} k\left(\cdot+E_{e}, E_{c}\right) q\left(E_{c}, E_{e}\right) d E_{e} d E_{c}
$$

It is readily observed that due to the affine construction of $k=\bar{P}, k \in L^{1}(\Omega)$ and $k \in L^{2}(\Omega)$ where

$$
\Omega=\Omega_{1} \times \Omega_{2} .
$$

The property that $k \in L^{1}(\Omega)$ is typical for convolution operators whereas $k \in L^{2}(\Omega)$ facilitates construction of a generalized Fourier basis for the operator. We employ this latter property in Section 3 to establish that $\mathcal{K}$ is a compact operator.

For implementation purposes, it is demonstrated in [18] that Gaussian quadrature can be employed to approximate the integrals, thus yielding the system

$$
[P(E)](t)=\sum_{i=1}^{N_{i}} \sum_{j=1}^{N_{j}} \nu\left(E_{c_{i}}, E_{e_{i}}\right)\left[\bar{P}\left(E_{e_{i}}+E ; E_{c_{j}}, \xi\right)\right](t) v_{i} w_{j}
$$

where $v_{i}$ and $w_{j}$ denote quadrature weights and $\nu: \mathbb{R}^{N_{i} \cdot N_{j}} \rightarrow \mathbb{R}$. To formulate (8) as a linear system, we define the $N_{i} \times N_{j}$ matrices $A(E)$ and $\Phi$ to have components

$$
\begin{aligned}
& {[A(E)]_{i j}=\left[\frac{E+E_{e_{j}}}{\eta}+P_{R} \delta\left(E ; E_{c_{i}}, E_{e_{j}}\right)\right] v_{i} w_{j}} \\
& {[Q]_{i j}=\nu\left(E_{c_{i}}, E_{e_{j}}\right) .}
\end{aligned}
$$

For $N=N_{i} \cdot N_{j}$, we define the $N \times 1$ vector $q$ and $1 \times N$ vector $a(E)$ by

$$
q=\operatorname{vec}(Q) \quad, \quad a(E)=[\operatorname{vec}(A(E))]^{T}
$$


where 'vec' denotes the vector concatenation of the respective matrices. The discretized polarization model (8) can then be formulated as the linear system

$$
P(E)=a(E) q .
$$

We note that $\eta$ is considered known and fixed in this formulation and is incorporated in $a(E)$.

\section{Compactness of the Polarization Operator}

In this section, we establish that the operator $\mathcal{K}$ given by $(7)$ is compact. As a prelude, we state the following theorem which is Theorem 5.24.8 from [10].

Theorem 1. Let $X$ and $Y$ be Banach spaces and let $\mathcal{K}_{N}: X \rightarrow Y, N=1,2, \ldots$, be a sequence of compact linear operators converging to a bounded linear operator $\mathcal{K}: X \rightarrow Y$; that is, $\left\|\mathcal{K}_{N}-\mathcal{K}\right\| \rightarrow 0$ as $N \rightarrow \infty$. Then $\mathcal{K}$ is a compact linear operator.

Remark 1. Consider the parameter space $\mathcal{Q}$ and observation space $\mathcal{Y}$ defined in (5) and (6). The integral operator given by (7) is then a compact operator. We establish this by demonstrating that $\mathcal{K}$ is the limit of a sequence of finite rank operators followed by the use of Theorem 1.

We first construct an orthonormal basis $\left\{\phi_{i}\right\}$ for $L^{2}(\Omega)$. It is illustrated in [10] that

$$
\varphi_{\ell}(s)=\frac{1}{\sqrt{E_{\max }-E_{\min }}} \exp \left[2 \pi i \ell \cdot \frac{s-E_{\min }}{\ell-E_{\min }}\right], \ell=0, \pm 1, \pm 2, \cdots
$$

forms an orthonormal basis for $L^{2}\left(\Omega_{1}\right)$. With an analogous basis definition for $L^{2}\left(\Omega_{2}\right)$, it follows that an orthonormal basis for $L^{2}(\Omega)$ is

$$
\phi_{\ell m}(s, t, v)=\varphi_{\ell}(s) \overline{\varphi_{m}(t, v)}
$$

which we re-index as $\left\{\phi_{i}\right\}$.

It follows that every $f \in L^{2}(\Omega)$ has the generalized Fourier series representation

$$
f=\sum_{i}\left\langle f, \phi_{i}\right\rangle \phi_{i}
$$

where $\langle\cdot, \cdot\rangle$ denotes the usual $L^{2}$ inner product. The norm representation

$$
\|f\|^{2}=\sum_{i}\left|\left\langle f, \phi_{i}\right\rangle\right|^{2}
$$

follows from Plancheral's theorem. Moreover, we can represent $\mathcal{K}$ and approximating finite-rank operators $\mathcal{K}_{N}$ by

$$
\begin{aligned}
& \mathcal{K} f=\sum_{i}\left\langle f, \phi_{i}\right\rangle \psi_{i} \\
& \mathcal{K}_{N} f=\sum_{i=1}^{N}\left\langle f, \phi_{i}\right\rangle \psi_{i}
\end{aligned}
$$

where $\psi_{i} \equiv \mathcal{K} \phi_{i}$. 
To establish the convergence $\mathcal{K} \rightarrow \mathcal{K}_{N}$, we note that

$$
\begin{aligned}
\left\|\mathcal{K} f-\mathcal{K}_{N} f\right\| & =\left\|\sum_{i \geq N+1}\left\langle f, \phi_{i}\right\rangle \psi_{i}\right\| \\
& \leq \sum_{i \geq N+1}\left|\left\langle f, \phi_{i}\right\rangle\right|\left\|\psi_{i}\right\| \\
& \leq\left[\sum_{i \geq N+1}\left|\left\langle f, \phi_{i}\right\rangle\right|^{2}\right]^{1 / 2}\left[\sum_{i \geq N+1}\left\|\psi_{i}\right\|^{2}\right]^{1 / 2} \\
& \leq\|f\|\left[\sum_{i \geq N+1}\left\|\psi_{i}\right\|^{2}\right]^{1 / 2}
\end{aligned}
$$

where the third inequality follows from the Schwartz inequality. Furthermore, we observe that

$$
\begin{aligned}
\sum_{i}\left\|\psi_{i}\right\|^{2} & =\sum_{i}\left[\int_{\Omega_{1}}\left|\mathcal{K} \phi_{i}(E)\right|^{2} d E\right] \\
& =\sum_{i} \int_{\Omega_{1}}\left|\iint_{\Omega_{2}} k\left(E+E_{e}, E_{c}\right) \phi_{i}\left(E_{c}, E_{e}\right) d E_{e} d E_{c}\right|^{2} d E \\
& =\int_{\Omega_{1}}\left[\sum_{i}\left|\iint_{\Omega_{2}} k\left(E+E_{e}, E_{c}\right) \phi_{i}\left(E_{c}, E_{e}\right) d E_{e} d E_{c}\right|^{2}\right] d E \\
& =\int_{\Omega_{1}}\left[\iint_{\Omega_{2}}\left|k\left(E+E_{e}, E_{c}\right)\right|^{2} d E_{e} d E_{c}\right] d E<\infty .
\end{aligned}
$$

The last step follows from Plancheral's theorem. The convergence of $\sum_{i}\left\|\psi_{i}\right\|^{2}$ implies that $\sum_{i \geq N+1}\left\|\psi_{i}\right\|^{2} \rightarrow 0$ as $N \rightarrow \infty$. Thus for $\varepsilon>0$, there exists $N_{\varepsilon}$ such that for $N>N_{\varepsilon}$,

$$
\left\|\mathcal{K}-\mathcal{K}_{N}\right\|=\sup _{f \neq 0} \frac{\left\|\mathcal{K} f-\mathcal{K}_{N} f\right\|}{\|f\|}<\varepsilon
$$

which establishes that

$$
\lim _{N \rightarrow \infty}\left\|\mathcal{K}-\mathcal{K}_{N}\right\|=0 .
$$

Since the range of $\mathcal{K}_{N}$ is finite, it follows that $\mathcal{K}_{N}$ is a compact operator. The compactness of $\mathcal{K}$ follows from Theorem 1 since it is the norm limit of a sequence of compact operators.

The compactness of $\mathcal{K}$ given by (7) is to be expected since it is a special case of a Hilbert-Schmidt operator which, in general, can be characterized as having an $L^{2}$ kernel. This is evidenced by the fact that the proof given here is a modification of that in [4] for Hilbert-Schmidt operators with kernels in $L^{2}\left(\mathbb{R}^{2 n}\right)$.

\section{Parameter Identification Problem}

For the operator $\mathcal{K}$ defined in (7), data $\widehat{P}$ corresponding to input fields $\widehat{E} \in L^{2}\left(E_{\min }, E_{\max }\right)$, and parameter space $\mathcal{Q}=L^{2}\left(\Omega_{2}\right)$, the parameter estimation problem can be formulated as follows: find $q \in \mathcal{Q}$ so that

$$
\mathcal{K} q=\widehat{P} .
$$

We note that (10) has a classical solution if and only if $\widehat{P} \in \mathcal{R}(\mathcal{K})$, where $\mathcal{R}(\mathcal{K})$ denotes the range of $\mathcal{K}$, which, in general, will not be true. Instead it is more reasonable to consider the least squares problem

$$
\min _{q \in \mathcal{Q}} T(q), T(q)=\frac{1}{2}\|\mathcal{K} q-\widehat{P}\|_{\mathcal{Y}}^{2} .
$$


However, because $\mathcal{K}$ is compact with infinite dimensional range, the Moore-Penrose inverse $\mathcal{K}^{\dagger}$ is discontinuous so that even (11) is ill-posed - see [3]. This motivates consideration of the augmented functional

$$
T_{\alpha}(q)=\frac{1}{2}\|\mathcal{K} q-\widehat{P}\|_{\mathcal{Y}}^{2}+\alpha \mathcal{J}(q)
$$

and the regularized least squares minimization problem

$$
\min _{q \in \mathcal{Q}} T_{\alpha}(q) .
$$

The regularization parameter $\alpha>0$ controls the tradeoff between goodness of fit to the data and stability whereas the penalty functional $\mathcal{J}$ provides stability and allows the inclusion of a priori information regarding the parameter $q$. One choice for $\mathcal{J}$ is the Tikhonov functional which we illustrate in the context of the discretized problem.

To formulate the finite-dimensional parameter estimation problem, we modify the linearly parameterized system (9) to reflect measured data. We define the $N_{i} \times N_{j}$ matrices

$$
\begin{aligned}
& {\left[A_{k}\right]_{i j}=\left[\frac{\widehat{E}_{k}+E_{e_{j}}}{\eta}+P_{R} \delta\left(\widehat{E}_{k} ; E_{c_{i}}, E_{e_{j}}\right)\right] v_{i} w_{j}} \\
& {[Q]_{i j}=\nu\left(E_{c_{i}}, E_{e_{j}}\right)}
\end{aligned}
$$

and vector concatenations

$$
q=\operatorname{vec}(Q) \quad, \quad a_{k}=\left[\operatorname{vec}\left(A_{k}\right)\right]^{T}
$$

so that $q$ and $a_{k}$ are respectively $1 \times N$ and $N \times 1$ where $N=N_{i} \cdot N_{j}$. Additionally, the $N_{d} \times 1$ vectors $\mathcal{P}$ and $\widehat{\mathcal{P}}$ are defined componentwise by

$$
[\mathcal{P}]_{k}=P\left(\widehat{E}_{k} ; q\right) \quad, \quad[\widehat{\mathcal{P}}]_{k}=\widehat{P}_{k}
$$

and the $N_{d} \times N$ matrix $A$ is defined row-wise by

$$
[A]_{k}=a_{k} .
$$

The discretized polarization model (8) can then be formulated as the linearly parameterized system

$$
\mathcal{P}\left(\widehat{E}_{k}\right)=A q .
$$

The unregularized least squares problem used to estimate $q=\nu \in Q=\mathbb{R}^{N_{i} \cdot N_{j}}$ given measurements $\left\{\left(\widehat{E}_{k}, \widehat{P}_{k}\right)\right\}, k=1, \ldots, N_{d}$ is the following:

$$
\begin{aligned}
& \min _{q \in Q} T(q), T(q)=\frac{1}{2}\|A q-\widehat{\mathcal{P}}\|^{2} \\
& \text { subject to } q_{i} \geq 0, j=1, \ldots, N .
\end{aligned}
$$

Here $\|\cdot\|$ denotes the Euclidean norm in $\mathbb{R}^{N}$. To incorporate Tikhonov regularization, we consider the minimization problem

$$
\begin{aligned}
& \min _{q \in Q} T_{\alpha}(q), T(q)=\frac{1}{2}\left\|A q-\widehat{\mathcal{P}}_{k}\right\|^{2}+\frac{\alpha}{2}\|q\|^{2} \\
& \text { subject to } q_{i} \geq 0, j=1, \ldots, N .
\end{aligned}
$$

Techniques for choosing $\alpha$ to avoid oversmoothing solutions as well as a solution algorithm for (16) can be found in Vogel [20]. 


\section{Validation Example}

To illustrate attributes of the least squares parameter estimation formulations (15) and (16) for estimating the $N=N_{i} \cdot N_{j}$ parameters $\left\{\nu\left(E_{c_{i}}, E_{e_{j}}\right)\right\}$, we consider the characterization of PZT5H. The unregularized model fits obtained with $N_{i}=N_{j}=24$ and $N_{i}=N_{j}=48$ using data from all seven hysteresis loops are plotted in Figure 2 whereas those obtained using the same quadrature limits in the regularized functional are given in Figure 3. Without regularization, the ill-posedness associated with inversion of the compact integral operator $\mathcal{K}$ yields increasingly inaccurate model predictions as discretization limits are increased. Regularization through the inclusion of the penalty term $\frac{\alpha}{2}\|q\|^{2}$ stabilizes the pseudoinverse by shifting singular values away from the origin thus yielding the highly accurate fit observed in Figure 3.

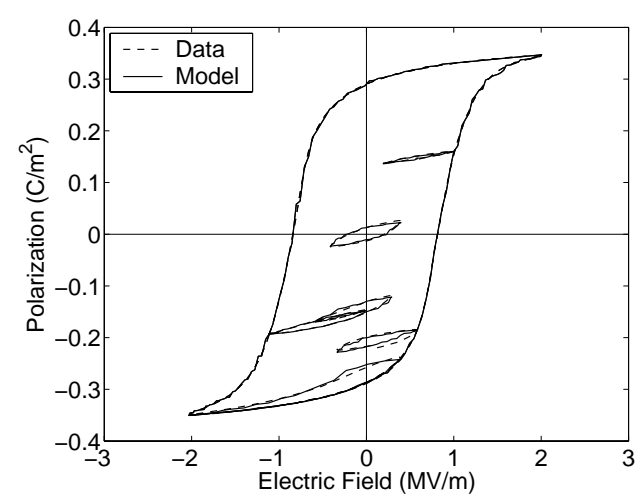

(a)

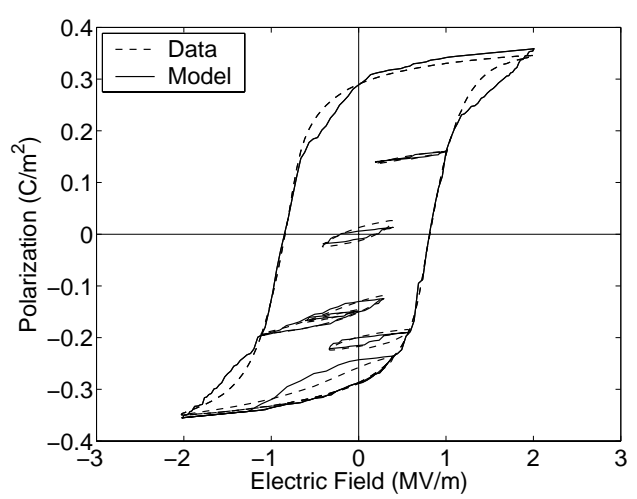

(b)

Figure 2: PZT5H data and model fit with general product density $\nu$ estimated using the unregularized functional (15) with data from all 7 loops. (a) $N_{i}=N_{j}=24(N=576)$, and (b) $N_{i}=N_{j}=48(N=2304)$.

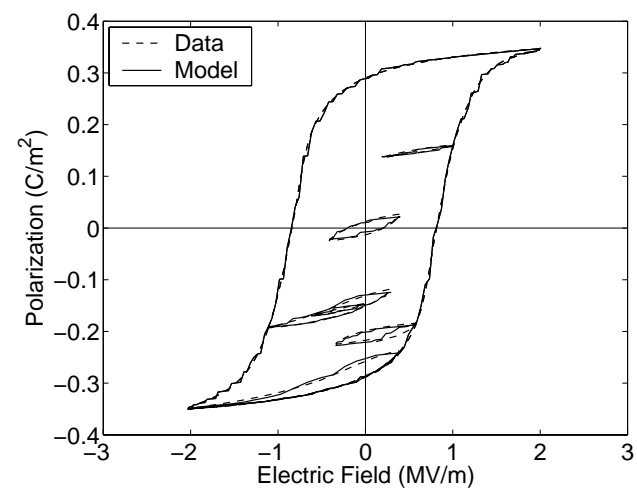

(a)

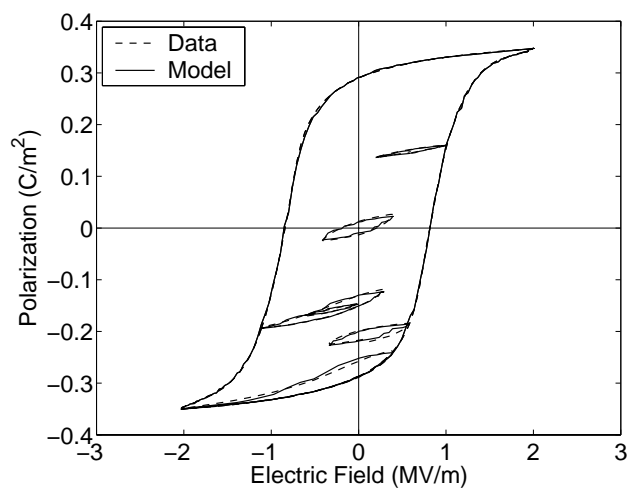

(b)

Figure 3: PZT5H data and model fit with general product density $\nu$ estimated using the regularized Tikhonov functional (16) with data from all 7 loops. (a) $N_{i}=N_{j}=24(N=576)$, and (b) $N_{i}=N_{j}=48(N=2304)$.

\section{Concluding Remarks}

In this paper we have developed a well-posed framework for estimating parameters in a model used to characterize the hysteretic and nonlinear field-polarization relation inherent to ferroelectric materials. The model is comprised of a compact integral operator with infinite-dimensional range so regularization is required to guarantee that the inverse problem is well-posed. It is illustrated through a fit to PZT5H data that Tikhonov regularization yields highly accurate model fits which retain stability as discretization limits are increased. 


\section{Acknowledgements}

This research was supported through the NSF grant CMS-009764 and the Air Force Office of Scientific Research under the grant AFOSR-F49620-01-1-0107.

\section{References}

[1] W.S. Galinaitis and R.C. Rogers, "Compensation for hysteresis using bivariate Preisach models," SPIE Smart Structures and Materials, 1997, Mathematics and Control in Smart Structures, San Diego, CA, 1997.

[2] P. Ge and M. Jouaneh, "Modeling hysteresis in piezoceramic actuators," Precision Engineering, 17, pp. 211$221,1995$.

[3] C.W. Groetsch, The Theory of Tikhonov Regularization for Fredholm Equations of the First Kind, Pitman, Boston, 1984.

[4] P.D. Hislop and I.M Sigal, Introduction to Spectral Theory with Applications to Schrödinger Operators, Springer-Verlag, New York 1996.

[5] R.V. Iyer, "Recursive estimation of the Preisach density function for a smart actuator," Proceedings of the SPIE, Smart Structures and Materials 2004, San Diego, CA, to appear.

[6] R.V. Iyer and P.S. Krishnaprasad, "On a low-dimensional model for ferromagnetism," Nonlinear Analysis: Theory, Methods and Application, submitted; preprint available at http://www. math.ttu.edu/ rvenkata.

[7] R.V. Iyer and M.E. Shirley, "Hysteresis identification with limited experimental data," IEEE Transactions on Magnetics, submitted; preprint available at http://www.math.ttu.edu/ rvenkata.

[8] J.E. Massad and R.C. Smith, "A domain wall model for hysteresis in ferroelastic materials," Journal of Intelligent Material Systems and Structures, 14(7), 2003, pp. 455-471.

[9] J.E. Massad, R.C. Smith and G.P. Carman, "A free energy model for thin-film shape memory alloys," Proceedings of the SPIE, Smart Structures and Materials 2003, Volume 5049, pp. 13-23, 2003.

[10] A.W. Naylor and G.R. Sell, Linear Operator Theory in Engineering and Science, Springer-Verlag, New York, 2000.

[11] D.C. Jiles and D.L. Atherton, "Theory of ferromagnetic hysteresis," Magnetism and Magnetic Materials, 61, pp. 48-60, 1986.

[12] M.E. Shirley and R. Venkataraman, "On the identification of Preisach measures," Proceedings of the SPIE, Smart Structures and Materials 2003, Modeling, Signal Processing, and Control, Vol. 5049, pp. 326-336, 2003.

[13] R.C. Smith, M.J. Dapino and S. Seelecke, "A free energy model for hysteresis in magnetostrictive transducers," Journal of Applied Physics, 93(1), pp. 458-466, 2003.

[14] R.C. Smith and C.L. Hom, "Domain wall theory for ferroelectric hysteresis," Journal of Intelligent Material Systems and Structures, 10(3), pp. 195-213, 1999.

[15] R.C. Smith and J.E. Massad, "A unified methodology for modeling hysteresis in ferroic materials," Proceedings of the 2001 ASME Design Engineering Technical Conferences and Computers and Information in Engineering Conference, Vol 6, Pt B, pp. 1389-1398, 2001.

[16] R.C. Smith and S. Seelecke, "An energy formulation for Preisach models," Proceedings of the SPIE, Smart Structures and Materials 2002, Volume 4693, pp. 173-182, 2002. 
[17] R.C. Smith, S. Seelecke, M.J. Dapino and Z. Ounaies, "A unified model for hysteresis in ferroic materials," Proceedings of the SPIE, Smart Structures and Materials 2003, Volume 5049, pp. 88-99, 2003.

[18] R.C. Smith, S. Seelecke, Z. Ounaies and J. Smith, "A free energy model for hysteresis in ferroelectric materials," Journal of Intelligent Material Systems and Structures, 14(11), pp. 719-739, 2003.

[19] X. Tan, R. Venkataraman and P.S. Krishnaprasad, "Control of hysteresis: Theory and experimental results," Smart Structures and Materials 2001, Modeling, Signal Processing and Control in Smart Structures, SPIE Vol. 4326, pp. 101-112, 2001.

[20] C.R. Vogel, Computational Methods for Inverse Problems, SIAM, Philadelphia, PA, 2002. 